\title{
Savoir traditionnel sur les plantes antipaludiques à propriétés analgésiques, utilisées dans le district de Bamako (Mali)
}

\author{
Dénou Aa, , Koudouvo Kb, Togola Aa, Haïdara Ma, Dembélé S.Ma, Ballo F.Na, Sanogo Ra, Diallo Da, \\ Gbeassor $M^{b, c}$. \\ a Département de Médecine Traditionnelle (DMT), Institut National de Recherche en Santé Publique (INRSP), Bamako, \\ Mali. \\ ' Laboratoire de Physiologie Animale et de Pharmacologie des Substances Naturelles, Faculté des Sciences, Université \\ de Lomé, Togo. \\ c Centre de Recherche et de Formation sur les Plantes Médicinales (CERFOPLAM), Université de Lomé, Togo. \\ ‘Correspondance : Dr Adama Dénou, DMT B.P.1746 Bamako. Tél. (00223)76309543, Email : adamadenou@yahoo.fr
}

Original submitted in on $4^{\text {th }}$ May 2016. Published online at www.m.elewa.org on 30 th April 2017 https://dx.doi.org/10.4314/iab.v112i1.3

\section{RÉSUMÉ}

Objectif : Le but de cette étude était de recenser les plantes utilisées dans la prise en charge traditionnelle de la douleur palustre.

Méthodologie et résultats : Les enquêtes ont été menées de février à mars 2011 dans le district de Bamako et le cercle de Kati. Les informations ont été collectées par une nouvelle méthode d'enquête ethnobotanique dénommée «Achat en Triplet de Recettes Médicinales (ATRM)» couplée à l'interview semi-structurée auprès de vingt quatre tradipraticiens de santé (herboristes et tradipraticiens de santé composés de dix sept femmes $(70,8 \%)$ et sept hommes $(29,2 \%)$. Au total 54 espèces médicinales appartenant à 52 genres de 21 familles ont été recensées. Les Fabaceae (ex-Caesalpiniaceae), les Rubiaceae, les Combretaceae et les Asteraceae sont les familles les plus représentées. Onze espèces ont été recensées par les deux méthodes à la fois. Mitragyna inermis et Anogeissus leiocarpus étaient les plus citées. Les recettes de la présente étude sont principalement préparées par décoction $(92,8 \%)$ et majoritairement administrées par voie orale $(52,6 \%)$. Les espèces recensées provenaient principalement des savanes, foret, champs de culture et de jachère. Mitragyna inermis (Willd.) O. Kuntze, Afrormosia laxiflora (Benth. ex Bak.) Harms, Parkia biglobosa (Jacq.) Benth, Pteleopsis suberosa Engl. \& Diels, Cassytha filiformis Linn., Tamarindus indica Linn., sont véritablement menacées dans leur biotope à cause de leur surexploitation en médecine traditionnelle et méritent d'être sauvegardées pour leur conservation aux générations futures.

Conclusion et application des résultats : La présente étude montre que les herboristes et tradipraticiens de santé de Bamako connaissent des plantes analgésiques utilisées dans la prise en charge traditionnelle du paludisme. Cette étude a aussi montré que certaines espèces sont menacées. Des investigations ultérieures sont nécessaires pour sélectionner certaines espèces identifiées puis vérifier in vivo leurs activités analgésiques.

Mots clés : Ethnobotanique, plantes antipaludiques, propriétés analgésiques, nouvelle méthode, Mali. 


\section{Denou et al., J. Appl. Biosci. 2017 Savoir traditionnel sur les plantes antipaludiques à propriétés}

analgésiques, utilisées dans le district de Bamako (Mali)

Traditional knowledge on antimalarial plants having analgesic properties, used in Bamako District (Mali).

\section{ABSTRACT}

Objective: The present study aimed to record analgesic plants used in the traditional management of pain related to malaria.

Methodology and results: Surveys were carried out from February to March 2011 in Bamako district and Kati area. Informations were collected by using a new method "Achat en Triplet de Recettes Medicinales (ATRM) and semi-structured interview (SSI). Twenty four health traditional practitioners (17 women and 7 men) either $70.8 \%$ and $29.2 \%$ were surveyed about the plant species used in traditional medicine for the management of pain related to malaria in Bamako district and Kati area. The name of the plants, the plant parts, the modes of preparation and the modes of administration of recipes were recorded. Cited plants were collected and identified at the Department of Traditional Medicine (DMT). The plants ecological status was also determined. In total 54 plant species belonging to 52 genera of 21 families were recorded. Fabaceae (ex-Caesalpiniaceae), Rubiaceae and Combretaceae families were the most cited. Eleven plants species were both collected by ATRM and SSI methods. Mitragyna inermis and Anogeissus leiocarpus were most cited. Recipes from our study were mainly prepared in decoction (92.8\%) and majority administrated by oral route $(52.6 \%)$. The collected species were proved mainly in savannah, forest, field crops, and fallow land. Mitragyna inermis, Afrormosia laxiflora, Parkia biglobosa, Pteleopsis suberosa, Cassytha filiformis, Tamarindus indica, were really threatened in their ecological sites related to over-exploitation in traditional medicine.

Conclusion and application of results: The present study showed us that herbalists and traditional healers have a good knowledge of antimalarial plants with analgesic properties. Our results showed also that few species are threatened. Further investigations are necessary to select some identified plants and then to check in vivo their analgesic activities.

Keywords: Ethnopharmacological study, antimalarial plants, analgesic properties, new method, Mali.

\section{INTRODUCTION}

Dans les pays africains, les plantes constituent, les principaux moyens médicamenteux pour des soins pratiques en santé publique. Les travaux de N'guessan et al. (2009) rapportent qu'en médecine traditionnelle, certains organes de plantes, telles que les feuilles, les écorces, les racines sont utilisées dans le traitement d'affections courantes comme la fièvre typhoïde, les diarrhées, le paludisme, la bronchite et l'hypofertilité. Dans le monde 3,4 milliards de personnes sont exposées au paludisme. En 2012, il y avait approximativement 207 millions de cas de paludisme au monde avec $80 \%$ de cas entrainant $90 \%$ de décès en Afrique. Les décès étaient surtout rencontrés chez les enfants de moins de 5 ans avec 77\% (WHO, 2013). Par ailleurs le paludisme continue à être une des causes de morbidité et de mortalité. Le paludisme est une maladie causée par les parasites du genre Plasmodium et est transmis par le moustique femelle du genre Anopheles qui agit comme un hôte et un vecteur du parasite. Les symptômes de la maladie incluent les maux de tête, la fièvre, la fatigue générale et les troubles gastro-intestinaux (Smyth, 1994). En Afrique où le paludisme est endémique, l'accès aux médicaments conventionnels est réduit dans les zones rurales et les pratiques culturelles restent encore importants (OMS, 2008). Par conséquent, $80 \%$ de la population utilise la médecine traditionnelle pour traiter le paludisme (Phillipson, 1994 ; Marsh et al, 1995 ; Geoffrey et Kirby, 1996 ; OMS, 2008). Au Mali, le paludisme est le motif de consultations le plus fréquent dans les centres de santé avec 1,6 millions de cas cliniques signalés en 2009 (PNLP, Mali, 2010). Dans 34,4 \% des cas la maladie affecte les enfants de moins de 5 ans. La mortalité due à cette affection est parmi les causes de décès les plus élevées chez les enfants de moins de 5 ans : 45,7 \% (Sacko et al., 2003). Plusieurs espèces de plantes ont été traditionnellement utilisées pour le traitement du paludisme (Srisilam et Versham, 2003). L'apparition de la résistance de Plasmodium falciparum aux 
multiples antipaludiques a été rencontrée dans beaucoup de pays y compris le Mali. Face à ce phénomène de résistance du parasite aux antipaludiques classiques, il est nécessaire de chercher d'autres molécules plus efficaces dans le traitement du paludisme. Des études ont montré que plus de 1200 plantes appartenant à 160 familles sont utilisées dans le traitement du paludisme (Willcox et Bodeker, 2004). L'enquête ethnobotanique est une importante phase dans l'identification, la sélection et le développement des molécules thérapeutiques issues des plantes médicinales (Idowu et al., 2010). Les premières recherches du Département de Médecine Traditionnelle (DMT) ont abouti à la mise sur le marché du Malarialß qui est un mélange de trois plantes Cassia occidentalis $\mathrm{L}$. (Caesalpinaceae), Lippia chevalieri Moldenke (Verbenaceae), Spilantes oleracea Jacq (Asteraceae) (Diallo et al., 2007). Dans le processus de recherche d'un nouveau phytomédicament antipaludique, des enquêtes auprès des ménages et

\section{MATERIEL ET METHODES}

Présentation de la zone d'étude: Cette étude s'est déroulée dans le district de Bamako (Mali) élargi au cercle de Kati. Le Mali est un pays de l'Afrique de l'ouest situé entre les latitudes ( 10 et $25^{\circ}$ Nord) et les longitudes (4 à $12^{\circ}$ Ouest) avec une superficie de $1.241 .238 \mathrm{~km}^{2}$. C'est un pays continental limité au nord par l'Algérie, au sud par la Côte d'Ivoire et la Guinée, à l'est par le Niger et le Burkina Faso, et à l'ouest par la Mauritanie et le Sénégal. Le climat est intertropical à caractère soudanosahélien nettement marqué par l'alternance d'une saison sèche qui dure six à neuf mois et d'une saison pluvieuse qui dure trois à six mois. On y distingue les zones climatiques suivantes : la zone sud soudanienne (précipitations supérieures à $1300 \mathrm{~mm}$ par an), la zone Nord - soudanienne (précipitations comprises entre 1300 et $700 \mathrm{~mm}$ par an), la zone sahélienne (précipitations comprises entre 700 et $200 \mathrm{~mm}$ par an) et la zone sud saharienne (précipitations inférieures à $200 \mathrm{~mm}$ par an). Les températures moyennes annuelles varient de 26 à $30^{\circ} \mathrm{C}$ et la pluviométrie moyenne annuelle est de 700 $\mathrm{mm}$. Ces données géographiques sont presque les mêmes dans le District de Bamako et le Cercle de Kati. Le District de Bamako est situé sur le $7^{\circ} 59^{\prime}$ de longitude ouest et le $12^{\circ} 40^{\prime}$ de latitude nord sur les deux rives du fleuve Niger : La rive nord située entre le fleuve et le Mont Manding dans la plaine alluviale de $15 \mathrm{~km}$. La rive sud, un des tradipraticiens de santé, ont abouti à Argemone mexicana L., (Papaveraceae), comme plante efficace pour le traitement du paludisme (Diallo et al., 2005 ; Graz et al., 2005). Des études de toxicité ont démontré une sécurité d'emploi du décocté des feuilles de Argemone mexicana (Sanogo et al., 2009). Des essais cliniques ont permis de confirmer l'efficacité du décocté des feuilles de Argemone mexicana dans la prise en charge du paludisme simple (Willcox et al., 2007, Graz et al., 2010). Pour une meilleure utilisation de Argemone mexicana, un nouveau phytomédicament dénommé "Sumafura, TIEMOKO BENGALY" a été mis au point sous forme de tisane et de sirop. Au Mali, aucun des travaux menés dans le domaine de la recherche antipaludique, n'a porté sur les plantes antipaludiques à propriétés analgésiques. L'objectif du présent travail est de documenter l'utilisation traditionnelle des plantes médicinales dans la prise en charge de la douleur liée au paludisme.

site de plus de 12.000 ha s'étendant de la zone aéroportuaire jusqu'au fleuve. D'une superficie de 180198 ha, Bamako appartient à la zone Nordsoudanienne avec une saison sèche qui dure de novembre à avril et une saison pluvieuse (l'hivernage) de mai à octobre. La température moyenne annuelle est de $27,6^{\circ} \mathrm{C}$ (extrêmes de 34,8 et $21^{\circ} \mathrm{C}$ ). La population est majoritairement composée de Bamanans (Bambaras).

Collecte des données: Pour la collecte des données, seize herboristes (douze femmes et quatre hommes) ont été visité dans huit marchés de Bamako pendant le mois de février 2011 pour acheter des plantes antipaludiques à propriétés analgésiques suivant l'Achat en Triplet de Recettes Médicinales (ATRM) (Koudouvo, 2009). Après l'achat, des informations sur les modes de préparation et d'utilisation, la voie d'administration et la durée du traitement ont été recueillies sur la recette. Les noms des plantes en langues locales ont été aussi demandés à l'herboriste. En plus de la méthode d'ATRM, huit tradithérapeutes dont cinq femmes et trois hommes ont été enquêtés. Ces tradithérapeutes habitent les villages de Diamoussabougou et de Falayan situés dans la commune rurale de Safo du cercle de Kati. Les interviews avec les tradipraticiens de santé volontaires ont été réalisées en utilisant un questionnaire semi-structuré. Les caractéristiques botaniques et ethnobotaniques portant 
sur les plantes collectées, leurs noms en langues locales, les organes utilisés, les modes de préparation et d'utilisation, la voie d'administration, sont demandés et notés. Les sites de cueillettes de Falayan et de Safo ont été aussi visités suivant l'ATRM pour collecter d'autres informations. Sur ces sites; le milieu de vie et le port des espèces recensées et retrouvées sont notés. Le mode de prélèvement et les parties prélevées sont également enregistrés afin d'évaluer les menaces que constituent ces prélèvements de plantes médicinales sur les diversités biologiques.

\section{Identification des plantes:}

- Identification botanique: Les plantes ont été identifiées au DMT et confirmées par la nomenclature

\section{RESULTATS ET DISCUSSION}

Cette étude a montré que les herboristes sont majoritairement des femmes. Cela infirme les résultats des études réalisées au Burkina Faso (Sanon et al., 2002) et au Mali (Togola et al., 2005) qui avaient trouvé que les hommes pratiquaient majoritairement cette tâche. Au total, nous avons recensé 54 espèces médicinales appartenant à 52 genres de 21 familles (Tableau 1) dont les Fabaceae sont les plus représentées avec 22,22\%. Cette famille est suivie des Rubiaceae et des Combretaceae respectivement représentées par 12,97\% et $11,11 \%$ (Figure 1). Mitragyna inermis (Willd.) 0. Kuntze était la plante la plus citée avec $16,22 \%$ suivie de Anogeissus leiocarpus (DC.) Guill. \& Perr (7,66\%). Onze espèces ont été recensées à la fois par les deux méthodes. Cette étude est la première dans son genre au Mali. Dans des études ethnobotaniques antérieures sur les plantes antipaludiques, les auteurs n'ont pas singularisé les espèces à activités analgésiques. Au Mali, des travaux sur la prise en charge du paludisme par les tradipraticiens de santé avaient collecté 102 recettes et en ont recensé 66 plantes antipaludiques (Diallo et al., 2007). En 2009, Asase et Oppong- Mensah ont collecté sur les marchés du Ghana, 29 espèces antipaludiques appartenant à 22 familles. Koudouvo, (2009) a recensé au Togo 137 espèces appartenant à 53 familles dont les plus représentées sont les Caesalpiniaceae, les Euphorbiaceae et les Rubiaceae. En Côte d'lvoire 32 de l'Angiosperms Phylogeny Group classification (APG III, 2009).

- Identification ethnobotanique : Les noms en langue locale des espèces sont également obtenus sur place dans la langue du tradipraticien. Les précisions ont été faites par Seydou M. Dembélé et NGolo Ballo tous deux exerçant au service ethnobotanique et des matières premières du DMT.

Analyse des données: Nous avons utilisé le logiciel Excel 2007 pour déterminer les fréquences des différentes informations collectées.

plantes antipaludiques ont été recensées par interview semi-structurée. Les Apocynaceae et les Euphorbiaceae étaient les plus représentées (Zirihi et al., 2010). Au Cameroun, Azadirachta indica, Mangifera indica, Psidium guajava, Cassia occidentalis, Khaya senegalensis, Tamarindus indica, Citrus limonum, Eucalyptus sp., Carica papaya et Cymbopogon citratus sont les plantes les plus utilisées pour la prise en charge traditionnelle du paludisme (Saotoing et al., 2011). Au Benin 82 espèces végétales appartenant à 78 genres de 43 familles étaient recensées où les Rubiaceae et les Caesalpiniaceae étaient les plus représentées (Yetein et al., 2013). En Guinée, 109 espèces végétales appartenant à 84 genres de 46 familles ont été recensées. Les plus citées étaient Vismia guineensis, Parkia biglobosa, Nauclea latifolia, Harungana madagascariensis, Terminalia macroptera, Crossopteryx febrifuga, Terminalia albida, Annona senegalensis, et Nauclea pobeguinii (Traoré et al., 2013). En République Démocratique du Congo, 46 plantes antipaludiques ont été recensées par interview semistructurée et les Asteraceae les plus représentées mais Azadiratcha indica and Cymbopognon citratus était la plus citée (Kasali et al., 2014). Une récente étude ethnopharmacologique réalisée en Cote d'Ivoire a recenser 26 plantes antipaludiques appartenant à 26 genres de 19 familles où les Euphorbiaceae étaient les plus représentées (Bla et al., 2015). 


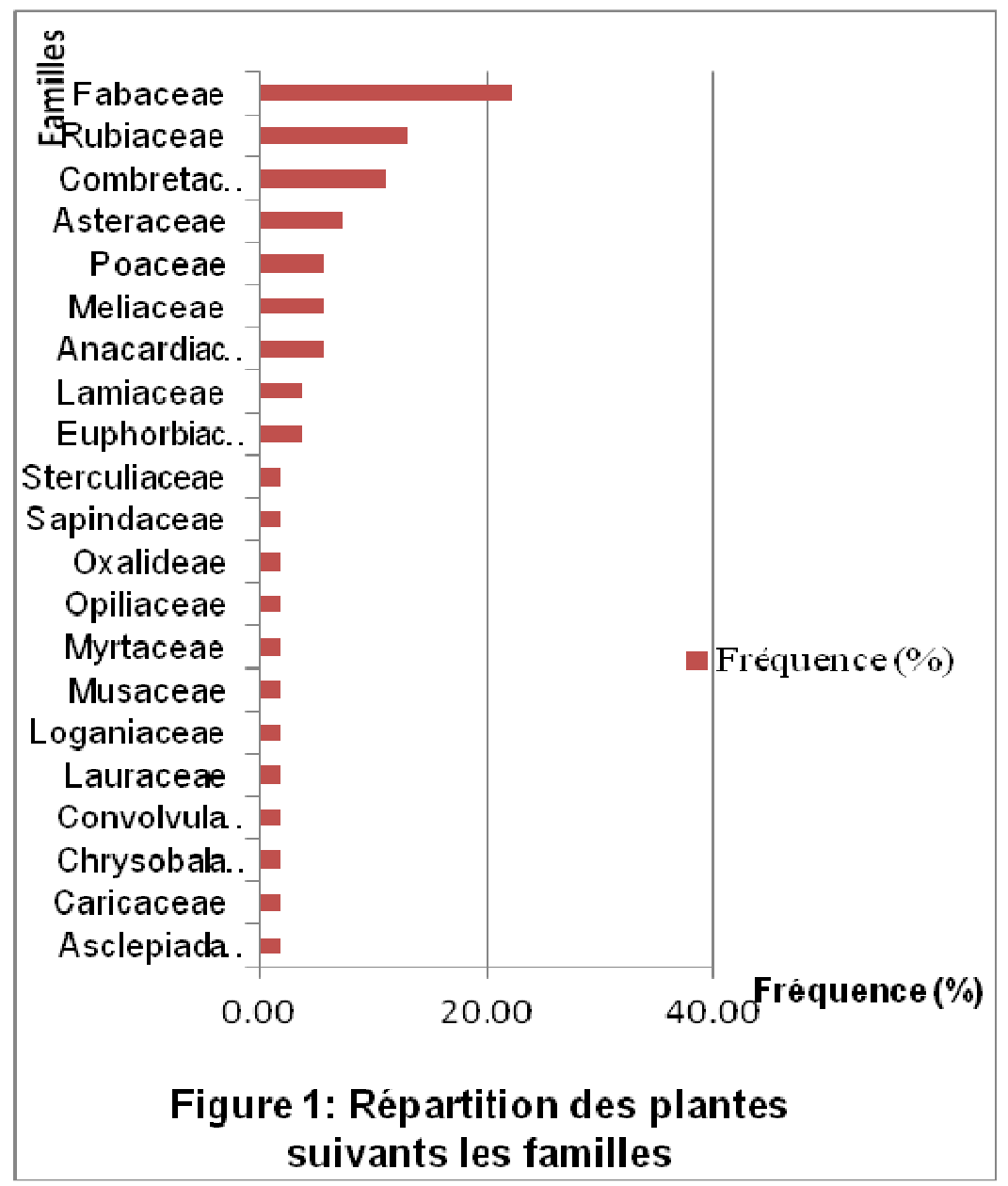

Sur les 54 espèces collectées, les arbres sont les plus cités avec $46,30 \%$ suivis des arbustes $22,22 \%$. Ces plantes sont surtout utilisées sous forme de : tige feuillée
$(65,52 \%)$, partie aérienne $(12,07 \%)$, écorce de tronc $(8,62 \%)$ et feuille $(6,90 \%)$ (Figure 2$)$.

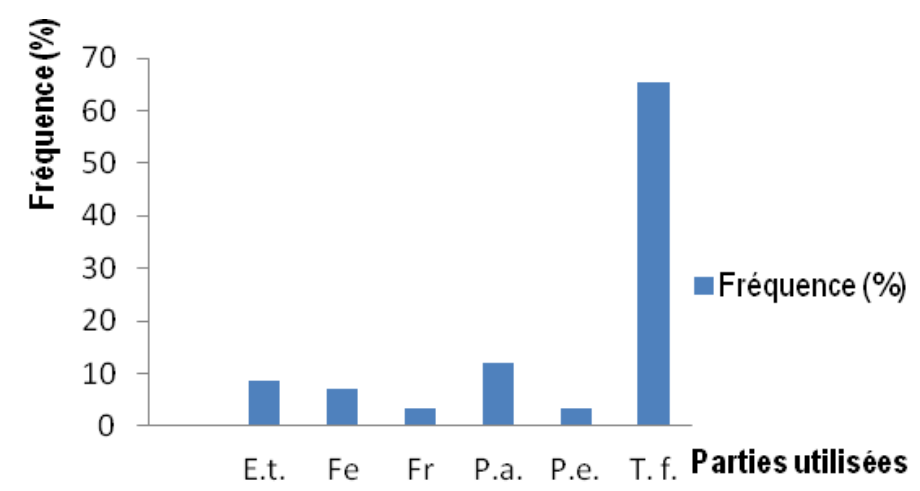

Figure 2: Frequence des parties utilisees

E.t.: Écorce de tronc, Fe: Feuille, Fr: Fruit, P.a.: Partie aérienne, P.e.: Plante entière, T.f.:Tige feuillée. 
Des travaux avaient signalé que les plantes antipaludiques étaient majoritairement des arbres ((Asase et al., (2005, 2010) ; Zirihi et al., 2010 ; Kasali et al., 2014). Quant à la partie utilisée, à notre connaissance aucun auteur n'a signalé la tige feuillée comme la plus utilisée mais plutôt la feuille (Caraballo et al., 2004; Asase et al., 2005; Koudouvo, 2009; Idowu et al., 2010; Saotoing et al., 2011; Traoré et al., 2013; Yetein et al., 2013; Kasali et al., 2014; Bla et al., 2015) et les racines (Ngarivhume et al., 2015). Par l'ATRM et I'ISS, la décoction ressort comme mode de préparation des recettes la plus utilisée avec $92,78 \%$ suivie de la macération (4,12\%). Ces recettes sont administrées à $52,58 \%$ par la voie orale. Nos résultats corroborent ceux de certains (Asase et al., (2005); Wondimu et al., (2007); Koudouvo (2009); Koudouvo et al., (2011a); Traoré et al., 2013; Yetein et al., 2013; Kasali et al et al., 2014; Bla et al et al., 2015) qui avaient trouvé que les plantes antipaludiques sont principalement préparées par décoction et administrées par voie orale. Sur les sites de cueillettes, les plantes antipaludiques à propriétés analgésiques poussent surtout sur les milieux comme la savane, la forêt, les champs de culture et de jachère. On y rencontre aussi des plantes cultivées, rudérales et semi aquatiques. Les mêmes milieux de vie étaient ressortis chez Koudouvo en 2006. Les plantes sont récoltées essentiellement suivant trois modes de prélèvement: prélèvement de l'organe, arrachement de la plante à ras le sol et arrachement total de la plante. Six espèces dont Mitragyna inermis (Willd.) O. Kuntze, Afrormosia laxiflora (Benth. ex Bak.) Harms, Parkia biglobosa (Jacq.) Benth, Pteleopsis suberosa Engl. \& Diels, Cassytha filiformis Linn. et Tamarindus indica Linn., sont véritablement menacées dans leur biotope à cause de leur surexploitation en médecine traditionnelle et méritent d'être sauvegardées pour leur conservation aux générations futures. Des travaux antérieurs ont signalé des menaces pour Lannea kerstingii A.Rich, Cola millenii K.Schum et Pavetta corymbosa (DC) F.N.Will dans la région maritime du Togo (Koudouvo, (2006, 2009). A partir de nos résultats, Mitragyna inermis et Sclerocarya birrea ont été sélectionnées par une technique d'élimination progressive qui est spécifique à l'ATRM; dans le but de vérifier in vivo leurs activités analgésiques. 
Tableau 1: Liste alphabétique des plantes antipaludiques à propriétés analgésiques recensées par les deux méthodes d'enquête au Mali

\begin{tabular}{|c|c|c|c|c|c|}
\hline $\mathbf{N}^{\circ}$ & NOMS SCIENTIFIQUES & FAMILLES & $\begin{array}{l}\text { NOMS LOCAUX } \\
\text { (BAMANAN) }\end{array}$ & $\begin{array}{l}\text { PARTIES } \\
\text { UTILISEES }\end{array}$ & $\begin{array}{l}\text { MODES DE PREPARATION/ VOIES } \\
\text { D'ADMINISTRATION }\end{array}$ \\
\hline 1 & Afrormosia laxiflora (Benth. ex Bak.) Harms & Fabaceae & Kolo kolo & Tige feuillée & Décoction/ Voies orale et corporelle \\
\hline 2 & Anogeissus leiocarpus (DC.) Guill. \& Perr & Combretaceae & nGalama & Tige feuillée & Décoction/ Voies orale et corporelle \\
\hline 3 & Azadirachta indica Linn. & Meliaceae & Mali jirinin, Sayi jirinin & Tige feuillée & Décoction/ Voies orale et corporelle \\
\hline 4 & Biophytum petersianum Klotzsch. & Oxalideae & Ju tugunin, Saki dawolo & Plante entière & Pulvérisation/Voies orale \\
\hline 5 & Bridelia micrantha (Hochst.) Baill & Euphorbiaceae & Sagan & Tige feuillée & Décoction/ Voies orale et corporelle \\
\hline 6 & Burkea africana Hook. & Fabaceae & Siri & Tige feuillée & Décoction/ Voies orale et corporelle \\
\hline 7 & Canthium sp. & Rubiaceae & Jama brubali & Tige feuillée & Décoction/ Voies orale et corporelle \\
\hline 8 & Carica papaya Linn. & Caricaceae & Manye, Papaye & Feuille & Décoction/ Voies orale et corporelle \\
\hline 9 & Cassia sieberiana DC. & Fabaceae & Sinjan & Tige feuillée & Décoction/ Voies orale et corporelle \\
\hline 10 & Cassytha filiformis Linn. & Lauraceae & Ala jon & Plante entière & Décoction/ Voies orale et corporelle \\
\hline 11 & Centaurea alexandrina Delile & Asteraceae & name ouani & Tige feuillée & Décoction/ Voies orale et corporelle \\
\hline 12 & Citrus aurantifolia (Christm. \& Panzer) Swingle & Rutaceae & Lemuru kumuni & Fruit & Décoction/ Voie orale \\
\hline 13 & Combretum glutinosum Perr. ex DC. & Combretaceae & Cangara & Tige feuillée & Décoction/ Voies orale et corporelle \\
\hline 14 & Combretum micranthum G. Don & Combretaceae & nGolobe & Tige feuillée & Décoction/ Voies orale et corporelle \\
\hline 15 & Combretum molle R.Br ex G. Don & Combretaceae & nanaka & Tige feuillée & Décoction/ Voie orale et corporelle \\
\hline 16 & Crossopteryx febrifuga (Afzel. ex G. Don) Benth. & Rubiaceae & Balembo & Tige feuillée & Décoction/ Voies orale et corporelle \\
\hline 17 & Daniellia oliveri (Rolfe) Hutch. \& Dalziel & Fabaceae & Sanan & Tige feuillée & Décoction/ Voies orale et corporelle \\
\hline 18 & Detarium senegalense J.F.Gmel. & Fabaceae & Tabakunba & Tige feuillée & Décoction/ Voies orale et corporelle \\
\hline 19 & Dicoma tomentosa Cassini & Asteraceae & Balaka wili & Plante entière & Décoction/ Voies orale et corporelle \\
\hline 20 & Dombeya sp. & Sterculiaceae & nTદfa & Tige feuillée & Décoction/ Voies orale et corporelle \\
\hline 21 & Feretia canthioïdes Hiern & Rubiaceae & Dioula sonkalani & Tige feuillée & Décoction/ Voies orale et corporelle \\
\hline 22 & Gardenia sokotensis Hutch & Rubiaceae & Farakoloci & Tige feuillée & Décoction/ Voie orales et corporelle \\
\hline 23 & Guiera senegalensis J.F. Gmel. & Combretaceae & Kunje & $\begin{array}{l}\text { Tige feuillée, } \\
\text { Fruit }\end{array}$ & Décoction/ Voies orale et corporelle \\
\hline 24 & Heeria insignis (Del.) O. Ktze & Anacardiaceae & Kala kari & Tige feuillée & Décoction/ Voies orale et corporelle \\
\hline 25 & Hyptis spicigera Lam. & Lamiaceae & Benefin jon & Plante entière & Décoction/ Voie corporelle \\
\hline 26 & Indigofera macrocalyx (G. Pers.) & Fabaceae & Cegana finsiki, Kuru kuru & Plante entière & Décoction/ Voies orale et corporelle \\
\hline 27 & Ipomoea repens Lam & Convolvulaceae & Ba Foroko faraka & Plante entière & Décoction/ Voies orale et corporelle \\
\hline 28 & Jatropha curcas Linn. & Euphorbiaceae & Baganin & Tige feuillée & $\begin{array}{l}\text { Infusion, Décoction / Voie } \\
\text { corporelle }\end{array}$ \\
\hline 29 & Leptadenia hastata (Pers.) Decne & Asclepiadaceae & nSonen & Tige feuillée & Décoction/ Voies orale et corporelle \\
\hline 30 & Mangifera indica Linn. & Anacardiaceae & Mangoro & $\begin{array}{l}\text { Tige feuillée, } \\
\text { Ecorce de tronc }\end{array}$ & Décoction/ Voies orale et corporelle \\
\hline
\end{tabular}


31 Mitragyna inermis (Willd.) Kuntze

32 Musa acuminata Colla

33 Opilia celtidifolia (Guill. \& Perr.) Endl.

4 Oxytenanthera abyssinica (A.Rich.) Munro

Parinari curatellifolia (Planch. ex) Benth.

Parkia biglobosa (Jacq.) Benth

7 Paullinia pinnata Linn.

Pennisetum pedicellatum Trinius

Piliostigma thonningii (Sch) Miln.Redh

0 Prosopis africana (Guill. \& Perr.)

1 Pseudocedrela kotschyi (Schweinf.) Harms

2 Psidium goyava Linn.

Pteleopsis suberosa Engl. \& Diels

4 Sarcocephalus latifolius (Smith) Bruce

Sclerocarya birrea (A. Rich.) Hochst.

Senna siamea (Lam.) H.S. Irwin \& Barneby.

Sorghum vulgare Pers.

Strychnos innocua Del.

Stylosanthes mucronata Wild.

Tamarindus indica Linn.

Trichilia emetica (Vahl)

2 Vernonia colorata Drake.

3 Vicoa leptoclada (Webb) Dandy

54 Vitex chrysocarpa Planch. ex Benth.

\begin{tabular}{|c|c|}
\hline $\begin{array}{l}\text { Rubiaceae } \\
\text { Musaceae } \\
\text { Opiliaceae } \\
\text { Poaceae } \\
\text { Chrysobalanaceae }\end{array}$ & \begin{tabular}{|l} 
Jun \\
Namasa \\
Korojoji \\
Bóó \\
nTama
\end{tabular} \\
\hline Fabaceae & 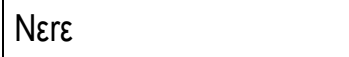 \\
\hline $\begin{array}{l}\text { Sapindaceae } \\
\text { Poaceae } \\
\text { Fabaceae } \\
\text { Fabaceae } \\
\text { Meliaceae } \\
\text { Myrtaceae } \\
\text { Combretaceae }\end{array}$ & $\begin{array}{l}\text { Faliwacan, Kurusaman } \\
\text { nGolo } \\
\text { nama } \\
\text { Gele } \\
\text { Zezan } \\
\text { Buyagi } \\
\text { Tereni }\end{array}$ \\
\hline Rubiaceae & Baro \\
\hline $\begin{array}{l}\text { Anacardiaceae } \\
\text { Fabaceae } \\
\text { Poaceae } \\
\text { Loganiaceae } \\
\text { Fabaceae } \\
\text { Fabaceae } \\
\text { Meliaceae } \\
\text { Asteraceae } \\
\text { Asteraceae } \\
\text { Lamiaceae }\end{array}$ & $\begin{array}{l}\text { nKuna } \\
\text { Kasia } \\
\text { non } \\
\text { nKantoro, nKankoronin } \\
\text { Segu fali } \\
\text { nTomi , nDomi } \\
\text { Surafinzan, Flafinzan } \\
\text { Ko safun } \\
\text { CEbleni kala } \\
\text { Koronifin }\end{array}$ \\
\hline
\end{tabular}

Tige feuillée

Feuille

Tige feuillée

Feuille

Tige feuillée

Tige feuillée,

Ecorce de tronc

Tige feuillée

Plante entière

Tige feuillée

Tige feuillée

Tige feuillée

Tige feuillée

Ecorce de tronc

Tige feuillée,

Ecorce de tronc

Ecorce de tronc

Tige feuillée

Feuille

Tige feuillée

Plante entière

Tige feuillée

Tige feuillée

Tige feuillée

Plante entière

Tige feuillée

Décoction/ Voies orale et corporelle

Décoction/ Voies orale et corporelle

Décoction/ Voies orale et corporelle

Décoction/ Voies orale et corporelle

Décoction/ Voie orale

Décoction/ Voies orale et corporelle

Décoction/ Voie orale et corporelle

Décoction/ Voie orale

Décoction/ Voie orale

Décoction/Voie corporelle

Décoction/ Voie orale

Décoction/ Voie orale

Macération/ Voie orale

Décoction, Macération/Voie orale

Décoction/ Voie orale

Décoction/ Voie orale

Décoction/ Voie orale

Décoction/ Voie orale

Décoction/ Voie orale

Décoction/ Voies orale et corporelle

Décoction/Voie orale

Décoction, Macération / Voie orale

Décoction/ Voies orale et corporelle

Décoction/ Voie orale 


\section{CONCLUSION}

La présente étude a montré que les tradipraticiens de santé du District de Bamako et ses environnants connaissent des plantes médicinales pour la prise en charge de la douleur liée au paludisme. Cependant cette étude a aussi révélé une menace de la biodiversité de certaines espèces qui méritent d'être sauvegardées pour

\section{REFERENCES}

Adjanohoun E.J, Ahyi A., Aké A.L., Dan Dicko L., Daouda H., Delmas M., de Souza S., Garba M., Guinko S., Koyong A., N'Golo D., Raynal J.L., Saadou M., 1980. Contribution aux études ethnobotaniques et floristiques au Niger, Médecine traditionnelle et pharmacopée ACCT. Paris.250p.

Adjanohoun E.J., Adjakidje V., Ahyi M.R.A., Ake A.L., Akpagana K., Chibon P., Al Hadji A., Eymé J., Garba M., Gassita J.N., Gbeassor M., Goudote E., Guinko S., HoundotoK., Houngnon P., Kéita A., Keoula Y., Klugu-Ocloo W.P., Siamevi K.M., Taffame K.K., 1986. Contribution aux études ethnobotaniques et floristiques au Togo. Agence de Coopération Culturelle et Technique. 526531.

Adjanohoun E.J., Adjakidje V., Ahyi M.R.A., Ake A.L., Akoegnou A., d'Almea J., Apovo F., Al Hadji A.F., Boukef K., Chadare M., Chibon P., Cusset G., Dramane K., Eymé J., Gassita. J.N., Gbaguidi M., Goudote E., Guinko S., Houngnon P., Lo I., Kéita A., Kiniffo H.V., Koné-Bamba D., Musampa Nseyya A., Saadou M., Sodogandji Th., de Souza S., Tchabi A., Zinsou Dossa C., Zohoun TH., 1989. Contribution aux études ethnobotaniques et floristique en République Populaire du Bénin. Collection«Médecine Traditionnelle et Pharmacopée». Agence de Cooperation Culturelle et Technique.pp.111, 339-405.

Akoègninou A., Van der Burg W.J,.Van Der Maesan L.J.G, Adjakidje V., Essou J.P.. Sinsin B, Yeomonhan H., 2006. Flore analytique du Bénin. Cotonou \& Wageningen, Backhuys Publishers, $1034 \mathrm{p}$.

APG III, 2009.La classification phylogénétique, modifiée de l'APG II (2003): An update of the Angiosperms. Phylogeny Group classification for the orders and families of flowerering plants. Botanical Journal of Linnean. Society, 141: 399436. leur conservation aux générations futures. Parmi les plantes recensées et identifiées certaines seront sélectionnées pour vérifier leurs propriétés analgésiques sur des modèles de laboratoire. Des études ultérieures seront entreprises dans le même sens sur le reste du territoire national.

Asase A., Oteng-Yeboah A.A., Odamtten G.T., Simmond M.S.J., 2005.Ethnobotanical study of some Ghanaian anti-malarial plants. Journal of Ethnopharmacology 99 ; 273-279.

Asase A., Oppong-Mensah G., 2009. Traditional antimalarial phytotherapy remedies in herbal markets in southern Ghana. Journal of Ethnopharmacology 126 (2009) 492-499.

Asase A., Akwetey G.A., Achel D.G., 2010. Ethnopharmacological use of herbal remedies for the treatment of malaria in the Dangme West District of Ghana. Journal of Ethnopharmacology (129) 367-376.

Betti J.L., 2002. Medicinal plants sold in Yaoundé market, Cameroon. African Study Monographs, 23(2): 44-64. Betti J.L., Mebere-Yemefa'a S.R, 2011. An ethnobotanical study of medicinal plants used in the Kalamaloue National Park, Cameroon. Journal of Medicinal Plants Research Vol. 5(8), pp. 1447-1458.

Bla K.B., Trebissou J.N.D, Bidie A.P., Assi Y. J., Zirihi G.N., Djaman A.J., 2015. Étude ethnopharmacologique des plantes antipaludiques utilisées chez les BaouléN'Gban de Toumodi dans le Centre de la Côte d'Ivoire. Journal of Applied Biosciences $85: 7775-7783$.

Brunel J.F., Schlz H., Hiekpo P., 1984. Flore analytique du Togo. Phanérogames. GTZ, Eschorn, 571p.

Caraballo A., Caraballo B., Rodríguez-Acosta A., 2004. Preliminary assessment of medicinal plants used as. Antimalarials in the southeastern Venezuelan Amazon Revista da Sociedade Brasileira de Medicina Tropical 37(2):186-188.

Diallo D., Graz B., Falquet J., Traoré A. K., Giani S., Mounkoro P.P., Berthé A., Sacko M., Diakité C., 2005. Malaria treatment in remote areas of Mali : use of modern and traditional medcines, patient outcome, The royal society of Tropical Medicine and Hygiène, Elvevier. Ltd, P7, 335.

Diallo D., Diakité C., Mounkoro P.P., Sangaré D., Graz B., Flaquet J., Giani S., 2007. Prise en charge 
du paludisme par les Thérapeutes Traditionnels dans les aires de Kendé (BANDIAGARA et de Finkolo (SIKASSO) au Mali. Mali Médical, p 1-8. Gbéassor M., Kossou Y., Amégbo K., de Souza C., Koumaglo K., Denké A., 1988a. Antimalarial testing of some African Medical plants. Experta Médica International Congress Series Edited by P. Kager and A.N.Polderman ; 810,346.

Gbéassor M., Kossou Y., Amégbo K., de Souza C., Koumaglo K., 1988b. Action de quelques plantes médicinales sur la croissance de Plasmodium falciparum in vitro. Médecine Traditionnelle et Pharmacopée (ACCT) 2,5-10.

Gbéassor M., Kossou Y., Amégbo K., de Souza C., Koumaglo K., 1989.Antimalarial effects of eigh African medicinal plants. Journal of ethnopharmacology, 25,115-118.

Gbéassor M., Kédjagni Y., Koumaglo K., de Souza C., Agbo K., Amégbo K., Aklikonou K., 1990. In vitro antimalarial activity six medicinal plants.Phytotherapy Research, 4 (3) :115-117.

Geoffrey C., Kirby, 1996. Medicinal plants and the control of protozoa disease with particular reference to malaria. Transactions of the Royal Society of Tropical Medicine and Hygiene 90, 605-609.

Graz B., Diallo D., Falquet J., Willcox M., Giani S., 2005. Screening of traditional herbal medicine: first, do a retrospective study, with correlation between diverse treatments used and reported patient outcome. Journal of Ethnopharmacology, 101:338-339.

Graz B., Willcox M., Diakite C., Falquet J., Dakouo F., Sidibe O., Giani S., Diallo D., 2009. Argemone mexicana decoction versus Artesunate/ Amodiaquine for the home-based management of malaria in Mali. Policy and public health implications. Transactions of the Royal Society of Tropical Medicine \& Hygiene 104:33-41.

Guyot M., 1992. Systématique des Angiospermes, (Référence à la flore du Togo). Agence de Coopération Culturelle et Technique. P 19-189.

Idowu O. A., Soniran O. T., Ajana O., Aworinde D.O., 2010. Ethnobotanical survey of antimalarial plants used in Ogun State, Southwest Nigeria. African Journal of Pharmacy and Pharmacology, Vol. 4(2) pp. 055-060.

Kasali F.M., Mahano A.O., Kadima N.J., Mpiana P.T., Ngbolua K.N., Tshibangu T.S.D., 2014. Ethnopharmacological Survey of Medicinal Plants Used against Malaria in Butembo City (D.
R. Congo). J. of Advanced Botany and Zoology $1,2348-7313$.

Koudouvo K., 2006. «Etude botanique et ethnobotanique sur les plantes Médicinales antipaludiques de la Région Maritime du Togo». Mémoire de DEA en Biologie de Développement, Option: Physiologie Pharmacologie, Faculté des Sciences, Université du Bénin, 63p.

Koudouvo K., 2009. Contribution à la recherche sur les plantes médicinales à propriété antipaludiques du Togo. Thèse de Doctorat de l'Université de Lomé en Biologie de Développement.Option: Ethnobotanique et Pharmacologie des Substances Naturelles, 182p.

Koudouvo K., Karou D.S, Kokou K., Essien K., Aklikokou K., Glitho I.A., Simpore, J., Sanogo R., De Souza C., Gbeassor M., 2011a. An ethnobotanical study of antimalarial plants in Togo Maritime Region. Journal of Ethnopharmacology (134) 183-190.

Koudouvo K., Karou D.S., Ilboudo D.P., Kokou K., Essien K., Aklikokou K., De Souza C., Simpore J., Gbeassor M., 2011b. In vitro antiplasmodial activity of crude extracts from Togolese medicinal plants. Asian Pacific Journal of Tropical Medicine 129-132.

Koumaglo K., Gbéassor M., Nikabou O., de Souza C., Werner W., 1992. Effects of three compounds extracted from Morinda lucida on Plasmodium falciparum . Planta Medica 58, 533-534.

Koumaglo K., Gbéassor M., Nikabou O., de Souza C.,2004. Activité de la Gedunine extraite de l'écorce du fruit de Azadirachta indica (Linn) sur Plasmodium falciparum in vitro. Phytotherapy journal, Vol 4. 78, 622-636.

Le Bras J., Musset L., Clain J., 2006. Antimalarial drug resistance. Médecine et Maladies Infectieuses 36, 401-405.

Marsh K., Foster D., Waruiru C., 1995. Indicators of lifethreatening malaria in African children. New England Journal of Medicine 332, 1399-1404.

Ngarivhume T.,. Van't Klooster C. I.E.A., de Jong J. T.V.M., Van der Westhuizen J. H., 2015. Medicinal plants used by traditional healers for the treatment of malaria in the Chipinge district in Zimbabwe. Journal of Ethnopharmacology 159 (2015) 224-237.

N'Guessan K, Tra B.F.H., Koné MW, 2009. Étude ethnopharmacologique de plantes antipaludiques utilisées en médecine traditionnelle chez les Abbey et Krobou 
d'Agboville (Côte d'Ivoire). Ethnopharmacologia, $44: 42-50$.

Odugbemi O.T., Akensulire R.O., Aibinu E.I., Fabeku O.P., 2007. Medicinal plants used for malaria therapy in Okeigbo, ondo State, Southwest of Nigeria. African Journal of Traditional, Complementary and Alternative Medicines 4, 191-198.

OMS, 2008. http://www.who.int.mediacentre/ factsheets/fs 13 .

Phillipson, J.D., 1994. Natural products as drugs. Transactions of the Royal Society of Tropical Medicine and Hygiene 88, 17-49.

PNLP Mali, 2010. « Semaine Africaine de Lutte contre le paludisme »Sacko M., Touré A., Tohon S., Udom B. B., Decosas J., Timmerman N., 2004. Mission consultative de faire reculer le paludisme (FRP) au Mali : actions essentielles pour soutenir l'atteinte des objectifs d'Abuja 10 14 novembre 2003. Mali RBM, Mission consultative rapport final.

Sangaré D., 2003. Etude de la prise en charge du paludisme par les thérapeutes traditionnels dans les cercles de santé de Kendié (Badiangara) et de Findolo A.C (Sikasso). Thèse de pharmacie, Bamako, 105p.

Sanogo R., Maïga A., Djimde A., Doumbia L., Guirou C., Diallo D., Doumbo O., 2009. Etude de la toxicité Sub- chronique du décocté de Argemone mexicana L. 15ème colloque sur la Pharmacopée et la Médecine Traditionnelles Africaines. Libreville.

Sanon S., Ollivier E., Azas N., Mahiou V., Gasquet M, Ouattara C.T., Nebie I., Traore A.S., Esposito F., Balansard G. Timon-David P., Fumoux F., 2002. Ethnobotanical survey and in vitro antiplasmodial activity of plants used in the traditional medicine in Burkina Faso. Journal of Ethnopharmacology 86, 143-147.

Saotoing P., used in traditional treatment of malaria in Cameroon. Journal of Ecology and the Natural Environment Vol. 3(3), pp. 104-117.

Smyth J.D., 1994. Animal Parasitology. Cambridge University Press, $549 \mathrm{pp}$.

Soh N.P., Benoit-Vical F., 2007. Are West African plants a source of future antimalarial drugs? Journal of Ethnopharmocology 114, 130-140.

Srisilam K., Versham C., 2003. Antimalarials of plant origin. In : Nishan, I., Khanu, A.(Eds.), Role of Biotechnology in medicinal and plants, vol. VII, pp. 17-47.
Tabuti S.R.T., 2008. Herbal medicines used in treatment of malaria in Budiope country, Uganda. Journal of Ethnopharmocology 116, 33-42.

Talisuna A.O., Bloland P., D'Alessandro U., 2004. History, dynamics, and public health importance of malaria parasite resistance. Clinical Microbiology Reviews 17, 235-254.

Togola A., Diallo D., Dembélé S., Barsett H., Paulsen S.B., 2005. Ethnopharmacological survey of different uses of seven medicinal plants from Mali (West Africa) in the regions Diola, Kolokani and Siby. Journal of Ethnobiology and Ethnomedicine 1, 7.

Traoré M.S., Baldé M.A., Diallo M.S.T., Baldé E.S., Diané S., Camara A., Diallo A., Balde A., Keïta A., Keita S.M., Oularé K., Magassouba F.B., Diakité I., Diallo A., Pieters L., Baldé A.M., 2013. Ethnobotanical survey on medicinal plants used by Guinean traditional healers in the treatment of malaria. Journal of Ethnopharmocology 150, 1145-1153.

Willcox M.L., Bodeker G., 2004. Traditional herbal medicines for malaria. British Medicinal Journal 329, 1156-1159.

Willcox M., Graz B., Falquet J., Sidibe O., Forster M., Diallo D., 2007. Argemone mexicana decoction for the treatment of uncomplicated falciparum malaria. Transaction of the Royal Society of Tropical Medicine and Hygiene 101:1190-1198.

Wondimu T., Asfaw Z., Kelbessa E., 2007. Ethnobotanical study of medicinal plants around Dheeraa' town, Arzizone, Ethiopia. Journal of Ethnopharmacology 112, 152-161.

WHO, 2013. World Malaria Report 2013. 〈http:// apps.whoint/iris/bitstream/10665/97008/1/97892 41564694_eng.pdf)(accessed on 3 April 2014).

Yetein M.H., Houessou L.G., Lougbégnon T.O., Teka O., Tente B., 2013. Ethnobotanical study of medicinal plants used for the treatment of malaria in plateau of Allada, Benin (West Africa). Journal of Ethnopharmacology 146 .154-163.

Zirihi G.N., N'guessan K, Etien Dibié T., Grellier P., 2010. Ethnopharmacological study of plants used to treat malaria, in traditional medicine, by Bete Populations of Issia (Côte d'Ivoire). Journal of Pharmaceutical Sciences and Research. 